\title{
Fighting against COVID-19: what operations research can help and the sense-and-respond framework
}

\author{
Tsan-Ming Choi ${ }^{1}$ (D)
}

Accepted: 28 January 2021

(c) The Author(s), under exclusive licence to Springer Science+Business Media, LLC part of Springer Nature 2021

\begin{abstract}
COVID-19 is affecting all walks of life. To deal with it, we need to make use of scientifically sound tools and models. Operations research (OR), as a well-established field which focuses on deploying analytical tools to solving decision making problems, comes to the rescue. In this paper, by examining the OR literature and practices related to pandemics (including COVID-19), we discuss what OR can help to tackle challenges under COVID19. We classify the literature into three stages, namely "before pandemic", "during pandemic" and "after pandemic". We examine the related literature and reveal the respective research areas and OR methods employed. Then, we propose a future research agenda. Finally, we establish the sense-and-respond OR framework regarding what specific actions should be taken to cope with COVID-19 from the perspectives of governments, healthcare and non-profit-making organizations, and businesses. We believe that the findings of this paper lay the solid foundation to stimulate further OR studies to combat COVID-19.
\end{abstract}

Keywords Sense-and-respond · COVID-19 · OR models $\cdot$ Framework

\section{Introduction}

"Let's hope the COVID-19 problem will be solved as soon as possible."

The author would like to express his sincere thanks to the editor and reviewers for their helpful and constructive comments.

Tsan-Ming Choi

jason.choi@polyu.edu.hk; tsanmingchoi@link.cuhk.edu.hk

1 Business Division, Institute of Textiles and Clothing, The Hong Kong Polytechnic University, Hung Hom, Kowloon, Hong Kong 


\subsection{Motivation and background}

COVID-19, the huge pandemic which is still troubling the whole world now, has started since winter 2019 (Araz et al. 2020). This virus outbreak has created lots of challenges and has killed more than one million people (as of 30 September 2020) ${ }^{1}$ and the figure is still growing. Statista.com mentions that "most major economies will lose at least $2.4 \%$ of their gross domestic product (GDP)" over $2020 .^{2}$ Nobody knows when the pandemic will be gone and scientists and medical researchers are still working on vaccines and finding the best treatment formulas for it.

Undoubtedly, COVID-19 creates severe threats to both business operations and human lives. It is hence a top priority agenda of all governments in the world. Governments commonly offer different kinds of subsidies to businesses as well as citizens. In some countries, measures have been imposed to limit cross-border travels. City lockdowns are also seen in some places; despite being able to reduce the spread of virus, it has a very negative impact on the economy. So, how to optimize social welfare becomes a big issue. As a remark, the social welfare under COVID-19 should include: (1) People's welfare (general public, managers, workers, and consumers), (2) business profits, (3) government's expenditure, and (4) health risk of medical staff and other people. This is an important objective function which deserves deeper investigations.

Operations research (OR), a field which focuses on using scientific models to enhance decision making in operations for profit making and non-profit making organizations, plays a crucial role. Traditionally, OR has an influential impact on healthcare operations including logistics (Kaur and Singh 2019; Modgil et al. 2020; Sun et al. 2020) for food and medicines, scheduling of staff members and capacity planning for treatment facilities, etc. (Rais and Viana 2010). In this paper, we review the OR literature and highlight what OR tools can help to deal with COVID-19 in three stages, namely "Stage 1: Before the pandemic", "Stage 2: During the pandemic" and "Stage 3: After the pandemic". We also focus on three main parties: (1) The governments, (2) the healthcare and non-profit making charity organizations, and (3) companies (i.e., business operations). As such, we establish a three-stage framework and an action plan on how to properly utilize OR models to address challenges created by COVID-19.

\subsection{Methodologies and contribution}

To find the OR models which are relevant to deal with COVID-19, we conduct a careful searching of the related literature. Our philosophy is on "quality" and "relevance". As a result, we focus on searching the well-estabilished journals which are mainstream in OR, including Annals of Operations Research, Computers and Operations Research, European Journal of Operational Research, International Transactions in Operational Research, Journal of the Operational Research Society, Operations Research, OR Letters. We also expand the scope to cover some journals in related areas such as in production (International Journal of Production Economics, International Journal of Production Research, Manufacturing and Service Operations Management, Management Science, Production and Operations Management) and logistics (e.g., Transportation

\footnotetext{
1 https://www.nature.com/articles/d41586-020-02762-y.

${ }^{2}$ https://www.statista.com/topics/6139/covid-19-impact-on-the-global-economy/.
} 
Research Part E). For searching, we search the keywords such as "COVID-19", "virus", "disease", "pandemic" in the paper titles. After searching, we manually review the contents and select the papers by relevance and review them. Some more papers are added based on the reviewed papers. Note that we do not aim to conduct an exhaustive and fully "scientific" survey of the whole literature. The focal point is on relevance and we also want to make use of the literature findings to enrich our understanding regarding what OR can help to fight against COVID-19, establish a promising research agenda, and build the respective sense-and-respond OR framework.

As a remark, there are also various papers which review the literature and discuss how academic research in OR may help address pandemic problems like COVID-19. To be specific, Queiroz et al. (2020) report a literature review on how pandemic affects supply chain operations. The authors find that in the current literature, as expected, "influenza" is the most widely explored topic. The authors also build a six-dimension framework dealing with COVID-19 from the perspective of operations management. Kaplan (2020) presents various adopted models from the literature which can be employed to conduct further research related to COVID-19. Silal (2020) highlights the "multidisciplinary" nature of OR for studying pandemic and diseases. The author discusses the topic from the angles of "disease biology", "intervention planning and implementation", "assessing economic feasibility of new strategies", "identifying opportunities for cost reductions in routine processes", and "informing health policy". Finally, the author proposes what OR can be used to effectively manage pandemic and diseases. Ivanov and Dolgui (2020) examine what OR models can help to dampen the supply chain "ripple effect" under COVID-19. The authors focus on examining the disruption literature and establish a taxonomy of measures to deal with COVID-19 and other similar infectious disease outbreaks. They also show that the "adaptation capabilities" dimension is most critical to support supply chain operations under pandemic such as COVID-19. Ivanov (2020a, b) applies the dynamic systems theory as well as the "viable supply chain" perspective to explore how supply chain systems can enhance their viability under COVID-19. The author highlights the fact that "resilience" is the most crucial dimension in achieving the viable supply chain system. The author also proposes various promising directions for further studies. Choi (2021) discusses risk control in logistics. The authors propose various research directions for risk analysis in the COVID-19 and post-COVID-19 ages. Baveja et al. (2020) propose a conceptual plan, which is based on the value chain model. Grounded on the "Theory of Constraints" (ToCs), the authors propose a specific plan which includes four measures, which should be implemented in a duration of more than 3 months. They also propose many tight measures such as "stopping all international, domestic passenger air and intercity bus/train travel" in order to help.

Similar to these review papers, we also build our framework and proposals via examining the literature. However, our proposed framework and focal point are very different from the above reviewed literature. In fact, even though the COVID-19 outbreak has started for a year, to the best of our knowledge, this study is the first paper which carefully examines the current OR literature and highlights what OR models can help address challenges brought by COVID-19 in the three stages (before, during and after pandemic) for the three-relevant parties. A sense-and-respond OR framework, with action plans, is also established which can provide guidance for practitioners and academics.

This paper is organized as follows. Section 2 reports the literature review. Section 3 presents the future research agenda. Section 4 develops the sense-and-respond OR framework. Section 5 concludes this paper. Figure 1 depicts this paper's structure. To enhance presentation, a list of notation is included in Table 1. 
Fig. 1 The paper's structure

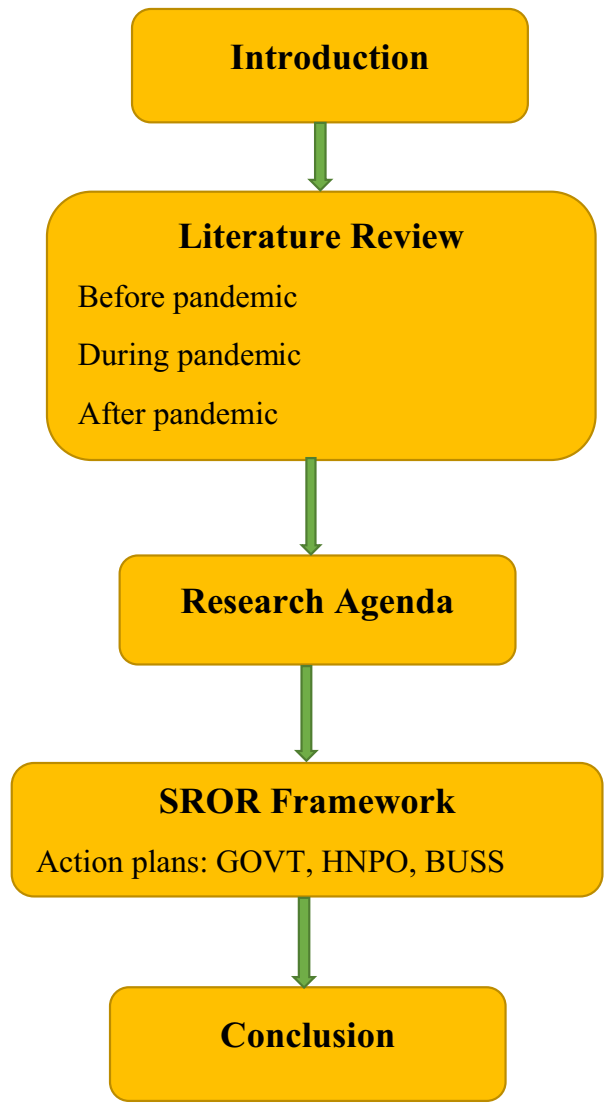

\section{Literature review}

In the following, we divide the literature with respect to the scope based on whether the explored operations challenges appear before, during or after the pandemic.

\subsection{Before pandemic}

Simulation has been widely used for forecasting and studying diseases (Davies 1994; Kunc and Kazakov 2013; Kang et al. 2018) and pandemic over the past decades. Before the pandemic happens, it is important to have a prediction regarding its seriousness. In the literature, Habbema et al. (1987) explore the "cost-effectiveness and cost-benefit" calculations for detecting diseases. The authors employ the simulation approach and indicate how real-world applications can be developed. In their established computer programs, ages and treatment stages are considered. They show the performance of their proposed models using data from Holland. Davies et al. (2003) conduct simulation analyses for the optimal healthcare models for "disease prevention and treatment". The authors try to uncover how "disease trends" and "treatment patterns" affect the optimal choice. They also explore costeffectiveness issues and argue that policy makers should employ the same set of assumptions when comparing among different models. To control pandemic, "mass-immunization 
Table 1 Notation table

\begin{tabular}{ll}
\hline Notation & Meaning \\
\hline OR & Operations research \\
COVID-19 & New coronas virus outbreak which started in winter 2019 \\
GOVT & Government \\
HNPO & Healthcare and non-profit-making organization \\
BUSS & Business \\
SROR & Sense-and-respond OR \\
SIM & Simulation \\
AMA & Analytical modeling analysis (economics analysis) \\
OPT & Optimization (computation-based) \\
RD & Research direction \\
RD1 & Optimization with social welfare under COVID-19 \\
RD2 & Location selection \\
RD3 & Facility capacity planning \\
RD4 & Scheduling of manpower \\
RD5 & Preventing diseases \\
RD6 & Multi-method approach and holistic studies \\
RD7 & Performance of innovative business models \\
RD8 & Using information technologies \\
\hline
\end{tabular}

clinics" (MCs) are a necessity. Beeler et al. (2014) study via "discrete-event simulation" the optimal staffing decisions at MCs. The authors argue that their paper is the first to model "flu transmission risk" at the MC, which is critical to decide the optimal staffing plan. Ventresca and Aleman (2014) develop a "randomized algorithm" to explore how virus outbreak can be controlled. The authors examine the "containment of pandemic disease" by vaccination. The authors further validate the performance of the proposed algorithm via exploring various common complex network models under several "cardinality constraints".

\subsection{During pandemic}

Once the pandemic has started, it will be critical to forecast its development so that the right reactive actions and policies can be made. In a classical study, Van Oortmarssen et al. (1981) investigate how a "micro-simulation program" can be developed to forecast effects of "mass screening" for diseases. It was a humble tool useful in the 1980s. Sun et al. (2009) build a "two-period multivariate Reed-Frost" model to capture the pandemic spread. The authors try to capture the exponential growth pattern of early pandemic spread. Uncertainties on the "number of initial infections", "spread of virus" and "drug efficacy" are considered. They prove the supermodularity feature of the game when "between-country infections" possess a low probability. Govindan et al. (2020) discuss how healthcare systems can best manage demand under COVID-19. The authors develop a useful and important data analytics tool (called "decision support system"). The system relies on medical doctors' inputs to build a "fuzzy inference system". This "fuzzy inference system" is the foundation for the data analytics tool. The authors demonstrate the performance of the developed tool by using data from several users in the real world. For conducting forecasting 
under COVID-19, Chen et al. (2020) make use of a "discrete-time Markov chain" model that puts uncertainties into the analysis. The authors believe that their proposed forecasting model is "flexible, robust, and accurate". The authors claim that their proposed model has been used in real world for medical workers and resource allocations. Nikolopoulos et al. (2020) study the growth rates of the COVID-19 pandemic using various scientific methods (including AI and statistics). The authors also predict excessive demand for different services and products. The authors argue that their findings can assist governments to set the proper measures for COVID-19 and other pandemics. Zhang et al. (2020a) adopt the "spatial-temporal model with network dynamics" to empirically study how people travel around the world in the presence of COVID-19. The authors make use of data from flights and explore the "pandemic correlations" across different countries in the presence of international restriction measures. The authors also investigate the correlations between people movement all around the world with respect to the target countries' pandemic situation.

Studying the pandemic in Africa, Büyüktahtakin et al. (2018) propose a novel "epidemics-logistics" (EL) model. The authors incorporate the spread of the epidemic as well as the related logistics challenges together into the analytical models. By formulating the EL model as an MIP optimization problem, the authors test the proposed model's applicability by conducting a retrospective test using data from countries like Guinea during the Ebola pandemic. The authors find that their model is valid and propose that more resources should be allocated to treatment and "isolation". After that, Liu et al. (2020) modify the model by Büyüktahtakin et al. (2018) by changing the capacity constraint and the authors actually explore a very similar problem.

Facing with expensive medical treatments, Mondschein et al. (2019) derive via stochastic dynamic programming the optimal rule which helps prescribe the treatment. The authors put into the optimization model critical factors of the patients such as age and treatment progress. The authors also derive a way to scientifically prioritize patients for treatment when budget is limited. Yaesoubi and Cohen (2011) propose a "generalized Markov model" to explore the spread of infectious diseases. The authors also establish a framework which helps derive the optimal dynamic policy for healthcare. One important contribution from this study is that, the proposed new model not only can be used to derive optimal dynamic policies for controlling "spreads of infectious diseases" but it is also efficient in terms of computational efficiency. As a result, the proposed dynamic policy is implementable as a real application. For patients infected of serious diseases such as COVID19, delayed diagnosis is a big deal. Fu et al. (2012) study via a "two-stage regression model" how different phases of a disease progression would relate to different risk factors (including delayed diagnosis). The authors further propose a novel "hazard-based regression model" to conduct further analyses. They conclude by highlighting the importance of including delayed diagnosis in the analytical models in order to yield a scientifically sound and valid result from risk analysis. Gunessee and Subramanian (2020) establish a framework for "supply chain decision making" and propose how it can be used to deal with problems such as COVID-19. Their research makes use of the "behavioral decision making theory". The authors interestingly uncover that "ambiguity" would affect optimal decision making in supply chain systems. They also find that there is a positive effect brought by "visibility".

Based on a reported real world case in Hong Kong, Choi (2020) analytically studies how the "bring-service-near-your-home" (BSNYH) operations can be a solution for small service providers to survive COVID-19. The author also highlights how the government may sponsor BSNYH in order to ensure its financial sustainability. The author further studies the potential application of blockchain to support the BSNYH operations. He theoretically 
derives the condition for establishing win-win as well as determines the "optimal safety technology investment" decision. Sun et al. (2014) build analytical models to study the optimal "patient and resource" allocation among hospitals. In the optimization model, the objective function includes minimization of both the total travel distance and maximum travel distance. One important feature of the model is that it can help forecast the occurrence of "resource shortage" during a pandemic. A real case study, with computational experiments and analyses, from the US is reported to demonstrate the applicability of the proposed model.

\subsection{After pandemic}

After COVID-19 is gone, the world will enter the post-COVID-19 era. Even though the exact situation is still largely unknown, a few studies have proposed and discussed some important points for this period of time. To be specific, in an insightful study, Ishida (2020) proposes various measures that need to be addressed in the post-COVID-19 era for supply chains selling tangible products. The author examines global supply chain operations for industries including auto-mobile, personal computers and home furnishing. The author studies these real cases via three theoretical viewpoints, namely "dynamic capability", "decentralization" and "concentration". Remko (2020) proposes a plan for building a more resilient supply chain for the "post COVID-19" era. The author interviews industrialists who are executives to learn about what they plan to do. Special attention is paid to how the standard supply chain management theories may be applicable to help. Working with Red Cross, Ekici et al. (2014) explore how a rapid response to a pandemic can be made by proposing a novel "disease spread" model. In their model, the authors study the optimal food distribution during a pandemic. To solve the problem, they develop heuristics. Using data from the US, the authors demonstrate how the model can be used and performed in practice. The authors further investigate the influence of "voluntary quarantine" on the demand for food and the corresponding distribution. Sharma et al. (2020) establish a theoretical scheme which helps to support "sustainable supply chain operations" during and after the COVID-19 outbreak. The authors conduct the "stepwise weight assessment ratio analysis" to uncover the critical factors for supporting the achievability of sustainable supply chain operations. The authors find that "supply chain viability" is one of the most critical factors.

Other than COVID-19, another important infectious disease is AIDS. Zanakis et al. (2007) study the impacts "social and economic determinants" of the pandemic of "AIDS" using empirical data from over 150 nations. The authors investigate 5 different measures. The authors further employ the data envelopment analysis (DEA) model, which would be helpful for governments to properly set the strategies for dealing with the spread of AIDS.

Table 2 shows a summary of the reviewed literature as well as the consolidated research areas in which OR can help deal with COVID-19.

\section{Research agenda}

Based on the reviewed literature and the observed practices under COVID-19, in the following, we discuss areas in which more research should be conducted. We call them "research directions" (RDs). 


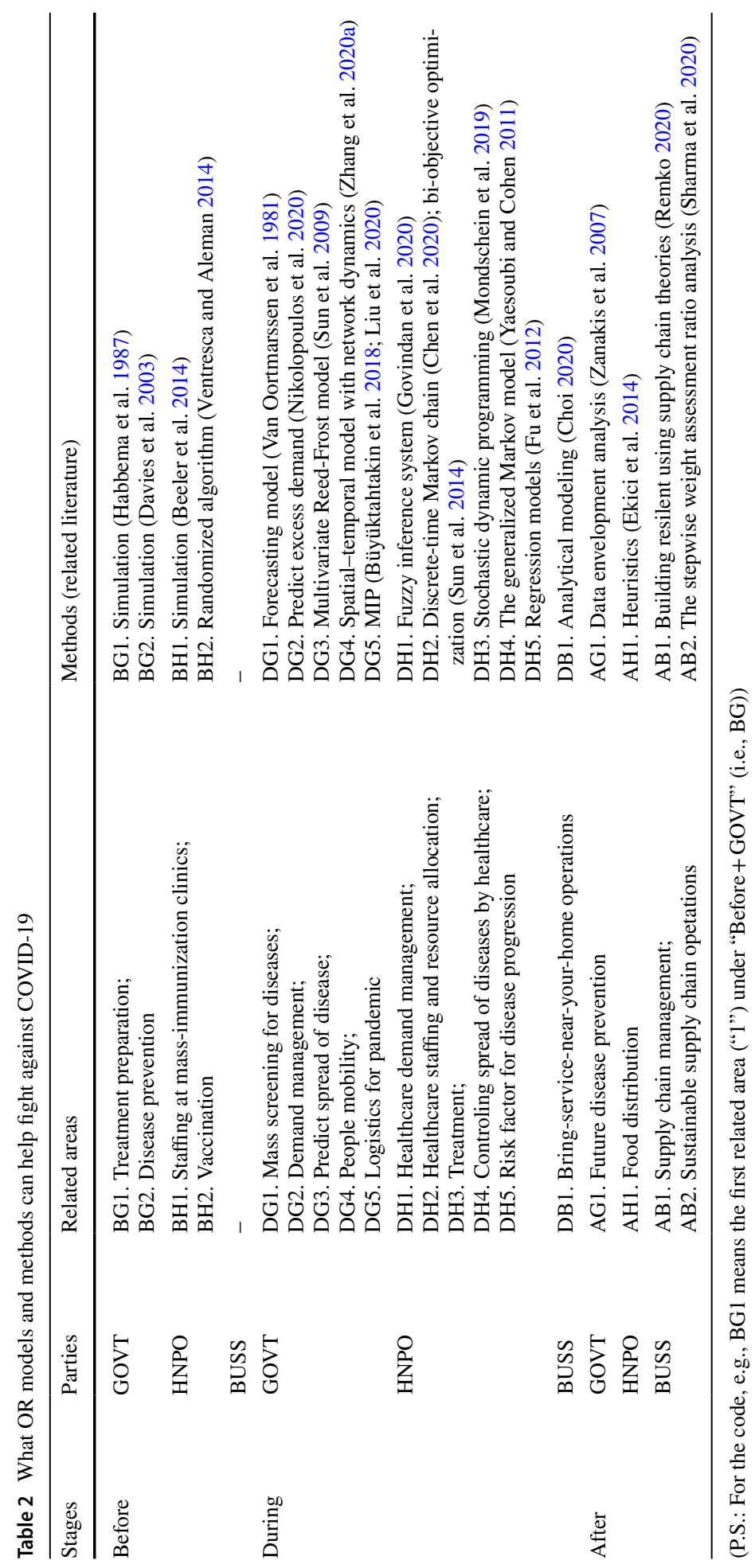




\subsection{Optimization with social welfare under COVID-19 (RD1)}

As we mentioned in Sect. 1, under COVID-19, social welfare should include: People's welfare (general public, managers, workers, and consumers), business profits, government's expenditure, and health risk of medical staff and people. This will be a very important matter because most current studies just focus on minimizing operations costs or travel distances. This is not sufficient. However, how to quantify and assign the right weighting to each item for this social welfare is an important issue for practical applications. How to build optimization models and solve them efficiently is another critical issue. Using stochastic programming, Özaltın et al. (2011) is an important reference for further studies in this proposed future research direction.

\subsection{Location selection (RD2)}

Under COVID-19, in order to avoid the infected patients spreading the virus around, the location for quarantine centers and hospitals must be carefully planned. In terms of the optimization problems and modeling approach, multi-criteria decision making (MCDM) should be a promising and appropriate OR tool. For references, Rahman and Smith (2000) is a very important paper which explores the "location-allocation" optimization problem. Chu and Chu (2000) is another study which examines optimal location and service allocation for hospitals. They lay the solid foundation for conducting further studies for this research direction.

\subsection{Facility capacity planning (RD3)}

Under COVID-19, the number of infected cases is stochastic. In many countries and places, the governments build ad-hoc and temporary "hospitals" and establish some temporary quarantine centers (Beeler et al. 2014). It is hence critical to decide the optimal capacity of these ad-hoc facilities, with respect to the projected number of infected cases. This will require a good forecasting tool as well as appropriate infectious-disease model to capture the real world scenarios specific to COVID-19. Moreover, whether flexibility is needed in order ensure facility capacity is available while not wasted should be seriously examined.

\subsection{Scheduling of manpower (RD4)}

Volunteers, patients and medical staff members (Cayirli and Veral 2003; Aickelin and Dowsland 2004; Bard and Purnomo 2005; Burke et al. 2008) are all involved with COVID19. In the literature (e.g., Rais and Viana 2010), we understand that manpower scheduling is critical for healthcare. For COVID-19, special features including the scale (larger than many other pandemics), infection rate (high infection), as well as the importance of volunteers, ${ }^{3}$ should be considered. Safety and infection risk of these related people should be considered, too. All these will make the manpower scheduling more complex because

\footnotetext{
${ }^{3}$ In Hong Kong, under COVID-19, many volunteers have helped for various critical activities like helping the elderly. However, their safety is also critically important. See "https://www.handsonhongkong.org/ safety_requirements" for an example of how a volunteering organization sets guidelines to help protect the volunteers under COVID-19.
} 
many additional factors need to be included in the objective functions as well as constraints. Of course, for companies, owing to COVID-19, many of them may no longer have the same demand level from the market. As such, how to dynamically schedule their staff members is a realistic challenge which should also be explored.

\subsection{Preventing diseases (RD5)}

COVID-19 will stop in the future. How to prevent it from happening again is a critical issue for governments and healthcare organizations. Moreover, how to prevent the pandemic of COVID-19 and other related diseases from appearing should be explored in the future. This relates to measures such as developing vaccine (Chick et al. 2008), establishing a sense-and-respond system to detect the probable occurrence of pandemic (see Sect. 4 for the proposed framework that we propose in this paper), as well as hygiene and health risk education. For vaccine, there are many prior studies which are related. An example is Wu et al. (2005), in which the authors investigate the optimal selection problem for flu vaccine. The recent paper by Martin et al. (2020) is another important reference for conducting analytically tractable future research for vaccine procurement.

\subsection{Multi-method approach and holistic studies (RD6)}

In the OR literature, most studies focus on exploring the decision making problems using one major research method/approach and focusing on one functional area. However, it will be more scientifically sound if we explore the problem using multiple methods (Choi et al. 2016) so that triangulation can be obtained. In addition, following the systems theory, it is usually insufficient if we just focus on improving one functional area. To obtain the globally optimal solution for the whole system, the holistic approach (e.g., using the system of systems theory Choi et al. 2019a) should be adopted in future studies. By doing so, we will also better understand the impacts from the systems perspective. This is especially critical for resource allocation problems (Xie et al. 2020). In particular, for the "before pandemic" part, our literature review indicates that most studies focus on simulation analyses. We propose that future research can be conducted by adding other methods. For example, theoretical modeling as well as statistical based quantitative empirical methods can be used together to enhance research rigor.

\subsection{Performance of innovative business models (RD7)}

Under COVID-19, many changes are observed. Online learning, teaching and meeting have become a "new normal". Related business operations hence arise. "Bring-service-toyour-home" (Choi 2020), "whatsapp shopping" service operations and many others (see Ravindran and Boh 2020; Amankwah-Amoah 2020) are also observed in practice. Owing to the innovative nature of these business models, deeper research should be conducted to enhance our understanding of their strengths and weaknesses. An important question to ask is: Whether these innovative models are just temporary (e.g., used during COVID-19) or sustainable in the long run deserves further investigations. 
Table 3 Future research on using OR to fight against COVID-19

\begin{tabular}{llllllllll}
\hline Stages & Parties & RD1 & RD2 & RD3 & RD4 & RD5 & RD6 & RD7 & RD8 \\
\hline Before & GOVT & A & A & A & A & A & A & & A \\
& HNPO & A & A & A & A & A & A & & A \\
& BUSS & & A & & A & & A & A & A \\
During & GOVT & A & A & A & A & A & A & & A \\
& HNPO & A & A & A & A & A & A & & A \\
& BUSS & & A & & A & & A & A & A \\
After & GOVT & A & A & A & A & A & A & & A \\
& HNPO & A & A & A & A & A & A & & A \\
& BUSS & & A & & A & & A & A & A \\
\hline
\end{tabular}

$(\mathrm{A}=$ Applicable $)$

\subsection{Using information technologies (RD8)}

To deal with COVID-19, many state-of-the-arts technologies can be used. For example, using big data (Choi et al. 2018a, b; Akter and Wamba 2019; Aras et al. 2020), artificial intelligence (Luo et al. 2019), blockchain (Choi 2019; Choi et al. 2019b; Choi and Luo 2019; Cai et al. 2020; Kalla et al. 2020) and wireless networks (Siriwardhana et al. 2020) can help to enhance traceability of patients and people suspected of having virus. Blockchain can also help to facilitate election and voting by keeping a permanent digital record and supporting secure digital operations. This will support the social distancing and city lockdown measures in many cities. It is crystal clear that a lot of future research can be conducted by studying how information technologies can be incorporated into real world operations to fight against COVID-19.

Table 3 shows a summary of the future research directions (RD1, RD2, ., RD8) as well as where they can fit into different stages related to pandemic, i.e., "before", "during" and "after" the pandemic, for different parties.

\section{The sense-and-respond OR framework}

From the literature review as well as the proposed future research agenda, it is evident that OR can help fights against COVID-19 in various areas. In this section, we propose a framework, called the "Sense-and-Respond OR (SROR) Framework" which can help to fight against pandemic similar to COVID-19. Figure 2 shows the SROR framework.

In the SROR framework, there are three modules, namely "before pandemic, "during pandemic", and "after pandemic". For each module, there are actions to be taken by three parties, namely the government (GOVT), healthcare and non-profit-making (charity) organization (HNPO), and business operations/companies (BUSS). The actions can be found in the action table (Table 4). To implement the actions, OR models and techniques are essential.

The philosophy of the SROR framework is that, the occurrence of pandemic is basically uncontrollable (i.e., it is random). However, it is critically important to get prepared and establish all the action plans before the pandemic occurs. Then, it is essentially important to detect (i.e., "sense") the occurrence of the pandemic as soon as possible and make 


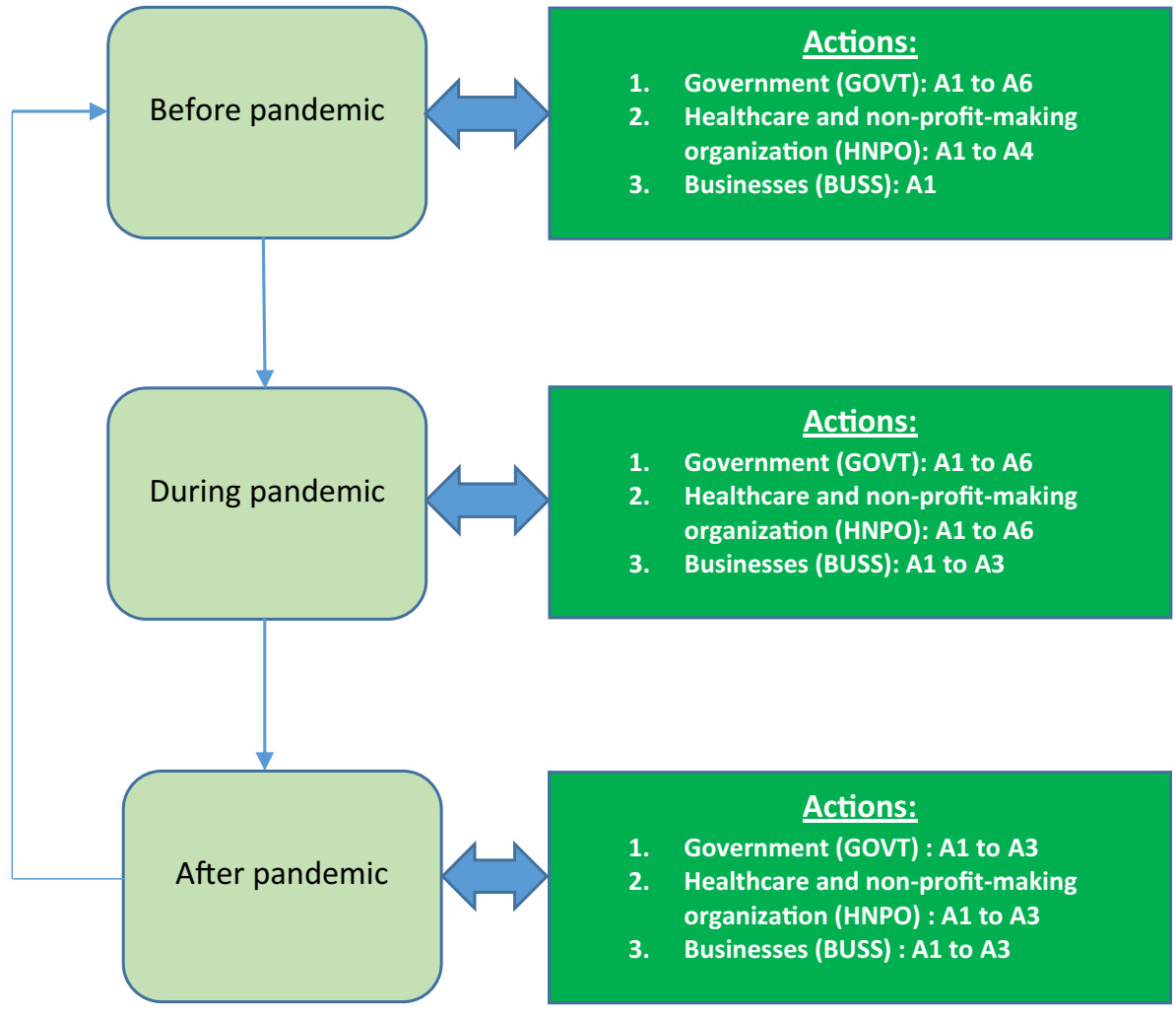

Fig. 2 The sense-and-respond OR (SROR) framework. (P.S.: The detailed actions (A1, .., A6) under each stage-party pair can be found in Table 4)

the right reactive decisions (i.e. "respond') in a timely manner. ${ }^{4}$ The SROR framework is very crucial to fight against pandemic and infectious diseases like COVID-19, and can be taken as a critical risk management related approach (Chiu and Choi 2016; Rahmani 2019; Zhang et al. 2020c).

Note that the SROR framework includes a feedback loop. When a pandemic is over, the gained experience and knowledge will be valuable to update action plans of the SROR framework so that future reactions will be improved.

\section{Conclusion}

As of today, there is no doubt that COVID-19 is affecting all walks of life. To properly deal with it, all academic disciplines should strive very hard to contribute. Operations research (OR) is no exception. In fact, OR, as a very well-established scientific field in both engineering and social sciences definitely can help. In fact, OR aims at applying analytical

\footnotetext{
${ }^{4}$ Responding in a timely manner is consistent with concepts such as quick response (Choi et al. 2018a, b; Li et al. 2020; Zhang et al. 2020b) and resilient supply chains (Fattahi et al. 2017; Elluru et al. 2019).
} 


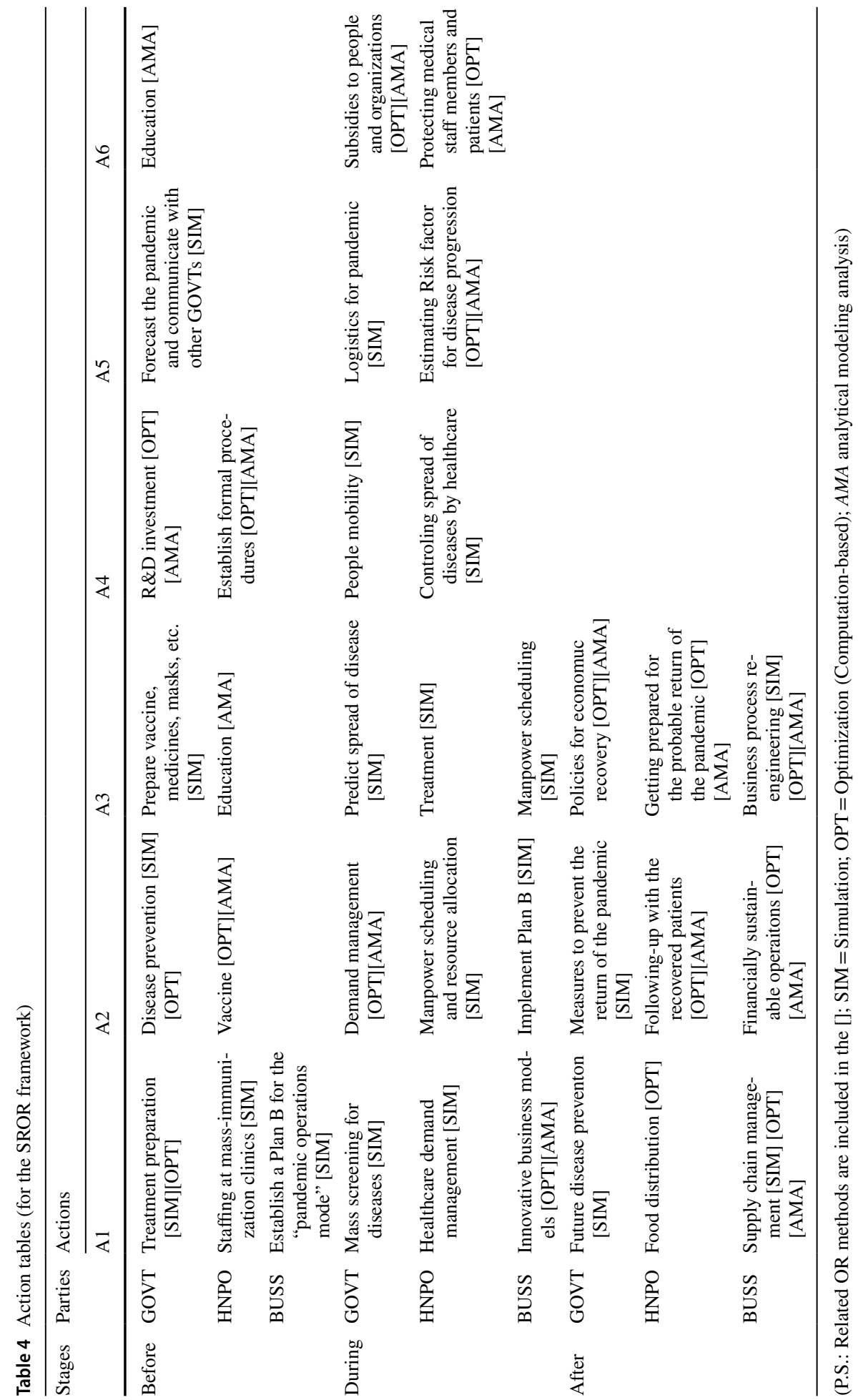


tools to solving decision making problems such as resource allocation, is naturally a discipline which can contribute a lot. Traditionally, OR helps with many healthcare operations problems such as (but not limited to) staff scheduling, medicine and vaccine allocation, humanitarian logistics for people and food, etc. (see Rais and Viana 2010). To fight against COVID-19, all these well-established techniques, tools and insights from OR should be valuable and applicable.

In this paper, by carefully examining the OR literature and practices related to pandemic, we have uncovered what OR can help to tackle challenges under COVID-19. We have classified the literature into three stages, namely "before pandemic", "during pandemic" and "after pandemic". We have examined the related literature and revealed the respective research areas that OR methods can be applied. We have further proposed a future research agenda which is specific to COVID-19. Finally, we have formally established the SROR framework regarding what OR can be used to deal with COVID-19 and the related operations problems.

We believe that this paper helps us better understand what OR can be used to fight against COVID-19. Hopefully, it would stimulate further OR studies and bring up more innovative ideas and methods to combat COVID-19.

All studies have limitations. For this paper, in searching for the related literature, we focus on quality as well as relevance rather than being very comprehensive. Some related papers are hence not covered. In particular, papers in medical and healthcare journals are not included. Future studies can hence be conducted to fill this gap.

\section{References}

Aickelin, U., \& Dowsland, K. A. (2004). An indirect genetic algorithm for a nurse-scheduling problem. Computers and Operations Research, 31(5), 761-778.

Akter, S., \& Wamba, S. F. (2019). Big data and disaster management: A systematic review and agenda for future research. Annals of Operations Research, 283, 939-959.

Amankwah-Amoah, J. (2020). Note: Mayday, Mayday, Mayday! Responding to environmental shocks: Insights on global airlines' responses to COVID-19. Transportation Research Part E: Logistics and Transportation Review, 143, 102098.

Aras, O., Choi, T. M., Olson, D., \& Salman, S. (2020). Role of analytics for operational risk management in the era of big data. Decision Sciences, 51(6), 1320-1346.

Bard, J. F., \& Purnomo, H. W. (2005). Preference scheduling for nurses using column generation. European Journal of Operational Research, 164(2), 510-534.

Baveja, A., Kapoor, A., \& Melamed, B. (2020). Stopping Covid-19: A pandemic-management service value chain approach. Annals of Operations Research, 289, 173-184.

Beeler, M. F., Aleman, D. M., \& Carter, M. W. (2014). A simulation case study to improve staffing decisions at mass immunization clinics for pandemic influenza. Journal of the Operational Research Society, 65, 497-511.

Burke, E. K., Curtois, T., Post, G., Qu, R., \& Veltman, B. (2008). A hybrid heuristic ordering and variable neighbourhood search for the nurse rostering problem. European Journal of Operational Research, $188(2), 330-341$.

Büyüktahtakin, I. E., Des-Bordes, E., \& Kibis, E. Y. (2018). A new epidemics-logistics model: Insights into controlling the Ebola virus disease in West Africa. European Journal of Operational Research, 265(3), 1046-1063.

Cai, Y. J., Choi, T. M., \& Zhang, J. (2020). Platform supported supply chain operations in the blockchain era: Supply contracting and moral hazards. Decision Sciences. https://doi.org/10.1111/deci.12475.

Cayirli, T., \& Veral, E. (2003). Outpatient scheduling in health care: A review of literaure. Production and Operations Management, 12(4), 519-549.

Chen, J., Fu, M. C., Zhang, W., \& Zheng, J. (2020). Predictive modeling for epidemic outbreaks: A new approach and COVID-19 case study. Asia-Pacific Journal of Operational Research, 37(3), 1-21. 
Chick, S. E., Mamani, H., \& Simchi-Levi, D. (2008). Supply chain coordination and influenza vaccination. Operations Research, 56(6), 1493-1506.

Chiu, C. H., \& Choi, T. M. (2016). Supply chain risk analysis with mean-variance models: A technical review. Annals of Operations Research, 240(2), 489-507.

Choi, T. M. (2019). Blockchain-technology-supported platforms for diamond authentication and certification in luxury supply chains. Transportation Research Part E: Logistics and Transportation Review, 128, 17-29.

Choi, T. M. (2020). Innovative "bring-service-near-your-home" operations under corona-virus (COVID19/SARS-CoV-2) outbreak: Can logistics become the Messiah? Transportation Research Part E: Logistics and Transportation Review, 140, 101961.

Choi, T. M. (2021). Risk analysis in logistics systems: A research agenda during and after the COVID19 pandemic. Transportation Research Part E: Logistics and Transportation Review, 145, 102190.

Choi, T. M., Cai, Y. J., \& Shen, B. (2019a). Sustainable fashion supply chain management: A system of systems analysis. IEEE Transactions on Engineering Management, 66(4), 730-745.

Choi, T. M., Cheng, T. C. E., \& Zhao, X. (2016). Multi-methodological research in operations management. Production and Operations Management, 25(3), 379-389.

Choi, T. M., \& Luo, S. (2019). Data quality challenges for sustainable fashion supply chain operations in emerging markets: Roles of blockchain, government sponsors and environment taxes. Transportation Research Part E: Logistics and Transportation Review, 131, 139-152.

Choi, T. M., Wallace, S. W., \& Wang, Y. (2018a). Big data analytics in operations management. Production and Operations Management, 27(10), 1868-1883.

Choi, T. M., Wen, X., Sun, X., \& Chung, S. H. (2019b). The mean-variance approach for global supply chain risk analysis with air logistics in the blockchain technology era. Transportation Research Part E: Logistics and Transportation Review, 127, 178-191.

Choi, T. M., Zhang, J., \& Cheng, T. C. E. (2018b). Quick response in supply chains with stochastically risk sensitive retailers. Decision Sciences, 49(5), 932-957.

Chu, S., \& Chu, L. (2000). A modeling framework for hospital location and service allocation. International Transactions in Operations Research, 7, 539-568.

Davies, R. (1994). Simulation for planning services for patients with coronary artery disease. European Journal of Operational Research, 72(2), 323-332.

Davies, R., Roderick, P., \& Raftery, J. (2003). The evaluation of disease prevention and treatment using simulation models. European Journal of Operational Research, 150(1), 53-66.

Ekici, A., Keskinocak, P., \& Swann, J. L. (2014). Modeling influenza pandemic and planning food distribution. Manufacturing and Service Operations Management, 16(1), 11-27.

Elluru, S., Gupta, H., Kaur, H., \& Singh, S. P. (2019). Proactive and reactive models for disaster resilient supply chain. Annals of Operations Research, 283, 199-224.

Fattahi, M., Govindan, K., \& Keyvanshokooh, E. (2017). Responsive and resilient supply chain network design under operational and disruption risks with delivery lead-time sensitive customers. Transportation Research Part E: Logistics and Transportation Review, 101, 176-200.

Fu, B., Wang, W., \& Shi, X. (2012). A risk analysis based on a two-stage delayed diagnosis regression model with application to chronic disease progression. European Journal of Operational Research, 218(3), 847-855.

Govindan, K., Mina, H., \& Alavi, B. (2020). A decision support system for demand management in healthcare supply chains considering the epidemic outbreaks: A case study of coronavirus disease 2019 (COVID-19). Transportation Research Part E: Logistics and Transportation Review, 138, 101967.

Gunessee, S., Subramanian, N. (2020). Ambiguity and its coping mechanisms in supply chains lessons from the Covid-19 pandemic and natural disasters. International Journal of Operations \& Production Management, published online.

Habbema, J. D. F., Lubbe, J. T. N., Van Oortmarssen, G. J., \& Van Der Maas, P. J. (1987). A simulation approach to cost-effectiveness and cost-benefit calculations of screening for the early detection of disease. European Journal of Operational Research, 29(2), 159-166.

Ishida, S. (2020). Perspectives on supply chain management in a pandemic and the post-COVID-19 era. IEEE Engineering Management Reivew, 40(3), 146-152.

Ivanov, D. (2020a). Predicting the impacts of epidemic outbreaks on global supply chains: A simulation-based analysis on the coronavirus outbreak (COVID-19/SARS-CoV-2) case. Transportation Research Part E: Logistics and Transportation Review, 136, 101922.

Ivanov, D. (2020b). Viable supply chain model: Integrating agility, resilience and sustainability perspectives-lessons from and thinking beyond the COVID-19 pandemic. Annals of Operations Research, published online. 
Ivanov, D., \& Dolgui, A. (2020). OR-methods for coping with the ripple effect in supply chains during COVID-19 pandemic: Managerial insights and research implications. International Journal of Production Economics, 232, 107921.

Kalla, A., Hewa, T., Mishra, R. A., Tlianttila, M., \& Liyanage, M. (2020). The role of blockchain to fight against COVID-19. IEEE Engineering Management Review, 48(3), 85-96.

Kang, H., Nembhard, H. B., Ghahramani, N., \& Curry, W. (2018). A system dynamics approach to planning and evaluating interventions for chronic disease management. Journal of the Operational Research Society, 69(7), 987-1005.

Kaplan, E. H. (2020). OM forum: COVID-19 scratch models to support local decisions. Manufacturing \& Service Operations Management, 22(4), 645-655.

Kaur, H., \& Singh, S. P. (2019). Sustainable procurement and logistics for disaster resilient supply chain. Annals of Operations Research, 283, 309-354.

Kunc, M., \& Kazakov, R. (2013). Competitive dynamics in pharmaceutical markets: A case study in the chronic cardiac disease market. Journal of the Operational Research Society, 64(12), 1790-1799.

Li, G., Li, L., Choi, T. M., \& Sethi, S. P. (2020). Green supply chain management in Chinese firms: Innovative measures and the moderating role of quick response technology. Journal of Operations Management, 66(7-8), 958-988.

Liu, M., Xu, X., Cao, J., \& Zhang, D. (2020). Integrated planning for public health emergencies: A modified model for controlling H1N1 pandemic. Journal of the Operational Research Society, 71(5), $748-761$.

Luo, S., Lin, X., \& Zheng, Z. (2019). A novel CNN-DDPG based AI-trader: Performance and roles in business operations. Transportation Research Part E: Logistics and Transportation Review, 131, 68-79.

Martin, P., Gupta, D., \& Natarajan, K. V. (2020). Vaccine procurement contracts for developing countries. Production and Operations Management, 29, 2601-2620.

Modgil, S., Singh, P.K., Foropon, C. (2020). Quality management in humanitarian operations and disaster relief management: A review and future research directions. Annals of Operations Research, published online.

Mondschein, S., Yankovic, N., Matus, O. (2019). Age-dependent optimal policies for hepatitis C virus treatment. International Transactions in Operational Research, published online.

Nikolopoulos, K., Punia, S., Schäfers, A., Tsinopoulos, C., \& Vasilakis, C. (2020). Forecasting and planning during a pandemic: COVID-19 growth rates, supply chain disruptions, and governmental decisions. European Journal of Operational Research, 290, 99-115.

Özaltın, O. Y., Prokopyev, O. A., Schaefer, A. J., \& Roberts, M. S. (2011). Optimizing the societal benefits of the annual influenza vaccine: A stochastic programming approach. Operations Research, 59(5), 1131-1143.

Queiroz, M.M., Ivanov, D., Dolgui, A., Wamba, S.F. (2020). Impacts of epidemic outbreaks on supply chains: mapping a research agenda amid the COVID-19 pandemic through a structured literature review. Annals of Operations Research, published online.

Rahman, S., \& Smith, D. K. (2000). Use of location-allocation models in health service development planning in developing nations. European Journal of Operational Research, 123, 437-452.

Rahmani, D. (2019). Designing a robust and dynamic network for the emergency blood supply chain with the risk of disruptions. Annals of Operations Research, 283, 613-641.

Rais, A., \& Viana, A. (2010). Operations research in healthcare: A survey. International Transactions in Operational Research, 18, 1-31.

Ravindran, T., \& Boh, W. F. (2020). Lessons from COVID-19: Toward a pandemic readiness audit checklist for small and medium-sized enterprises. IEEE Engineering Manageent Review, 48(3), 55-62.

Remko, H. (2020). Research opportunities for a more resilient post-COVID-19 supply chain-closing the gap between research findings and industry practice. International Journal of Operations \& Production Management, published online.

Sharma, M., Luthra, S., Joshi, S., Kumar, A. (2020). Developing a framework for enhancing survivability of sustainable supply chains during and post-COVID-19 pandemic. International Journal of Production Research, published online.

Silal, S.P. (2020). Operational research: A multidisciplinary approach for the management of infectious disease in a global context. European Journal of Operational Research, published online.

Siriwardhana, Y., Alwis, C. D., Gur, G., Ylianttila, M., \& Liyanage, M. (2020). The fight against the COVID-19 pandemic with 5G technologies. IEEE Engineering Management Review, 48(3), 72-84.

Sun, L., DePuy, G. W., \& Evans, G. W. (2014). Multi-objective optimization models for patient allocation during a pandemic influenza outbreak. Computers \& Operations Research, 51, 350-359.

Sun, P., Yang, L., \& De Véricourt, F. (2009). Selfish drug allocation for containing an international influenza pandemic at the onset. Operations Research, 57(6), 1320-1332. 
Sun, X., Chung, S. H., \& Ma, H. L. (2020). Operational risk in airline crew scheduling: do features of flight delays matter? Decision Sciences, 51(6), 1455-1489.

Van Oortmarssen, G. J., Habbema, J. D. F., Lubbe, J. T. N., de Jong, G. A., \& van der Maas, P. J. (1981). Predicting the effects of mass-screening for disease-A simulation approach. European Journal of Operational Research, 6(4), 399-409.

Ventresca, M., \& Aleman, D. (2014). A randomized algorithm with local search for containment of pandemic disease spread. Computers \& Operations Research, 48, 11-19.

Wu, J. T., Wein, L. M., \& Perelson, A. S. (2005). Optimization of influenza vaccine selection. Operations Research, 53(3), 456-476.

Xie, J., Zhuang, W., Ang, M., Chou, M.C., Luo, L., Yao, D.D. (2020). Analytics for hospital resource planning - Two case studies. Production and Operations Management, published online

Yaesoubi, R., \& Cohen, T. (2011). Generalized Markov models of infectious disease spread: A novel framework for developing dynamic health policies. European Journal of Operational Research, 215(3), 679-687.

Zanakis, S. H., Alvarez, C., \& Li, V. (2007). Socio-economic determinants of HIV/AIDS pandemic and nations efficiencies. European Journal of Operational Research, 176(3), 1811-1838.

Zhang, C., Qian, L.X., Hu, J.Q. (2020a). COVID-19 Pandemic with human mobility across countries. Journal of the Operations Research Society of China, published online.

Zhang, J., Choi, T. M., \& Cheng, T. C. E. (2020b). Stochastic production capacity: A bane or a boon for quick response supply chains. Naval Research Logistics, 67(2), 126-146.

Zhang, J., Sethi, S. P., Choi, T. M., \& Cheng, T. C. E. (2020c). Supply chains involving a mean-varianceskewness-kurtosis newsvendor: Analysis and coordination. Production and Operations Management, $29(6), 1397-1430$.

Publisher's Note Springer Nature remains neutral with regard to jurisdictional claims in published maps and institutional affiliations. 